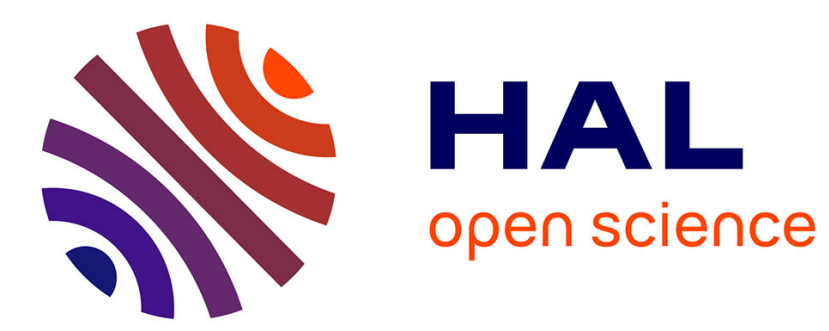

\title{
CENTRES COLORÉS DUS A DES DÉFAUTS CATIONIQUES CRÉÉS PAR DES IONS DE HAUTE ÉNERGIE DANS LiF ET KCl
}

\author{
P. Thévenard, A. Perez, J. Davenas, C. Dupuy
}

\section{To cite this version:}

P. Thévenard, A. Perez, J. Davenas, C. Dupuy. CENTRES COLORÉS DUS A DES DÉFAUTS CATIONIQUES CRÉÉS PAR DES IONS DE HAUTE ÉNERGIE DANS LiF ET KCl. Journal de Physique Colloques, 1973, 34 (C9), pp.C9-289-C9-293. 10.1051/jphyscol:1973951 。 jpa-00215426

HAL Id: jpa-00215426

https://hal.science/jpa-00215426

Submitted on 1 Jan 1973

HAL is a multi-disciplinary open access archive for the deposit and dissemination of scientific research documents, whether they are published or not. The documents may come from teaching and research institutions in France or abroad, or from public or private research centers.
L'archive ouverte pluridisciplinaire HAL, est destinée au dépôt et à la diffusion de documents scientifiques de niveau recherche, publiés ou non, émanant des établissements d'enseignement et de recherche français ou étrangers, des laboratoires publics ou privés. 


\title{
CENTRES COLORÉS DUS A DES DÉFAUTS CATIONIQUES CRÉÉS PAR DES IONS DE HAUTE ÉNERGIE DANS LiF ET KCl
}

\author{
P. THÉVENARD, A. PEREZ, J. DAVENAS et C. H. S. DUPUY \\ Département de Physique des Matériaux, associé au CNRS \\ Université Claude-Bernard, Lyon 1, 43, bd du 11-Novembre-1918, 69621 Villeurbanne, France
}

\begin{abstract}
Résumé. - La création et l'évolution des centres chargés dans $\mathrm{LiF}$ et $\mathrm{KCl}$ bombardés avec des particules de haute énergie mettent en évidence des mécanismes de recombinaison différents de ceux observés dans des cristaux irradiés avec des rayonnements essentiellement ionisants. Ceci peut être expliqué par l'existence de défauts d'origine cationique créés par chocs élastiques et piégeant les électrons de neutralité de ces centres chargés. L'agglomération de ces défauts cationiques par traitement thermique fait apparaittre des colloïdes métalliques et des bulles d'halogène en interaction. La position et l'évolution des bandes d'absorption associées aux colloïdes et aux bulles conduisent à envisager l'existence d’hydrogène à létat métallique. Cette hypothèse semble se vérifier dans le cas de LiF irradié avec des protons.
\end{abstract}

\begin{abstract}
The creation and the evolution of charged centres in LiF and $\mathrm{KCl}$ bombarded with high energy particies exhibit some recombination mechanisms different from those observed in crystals irradiated with ionizing radiations. This can be explained by the presence of cationic defects trapping the electrons. The aggregation of the cationic defects by thermal annealings shows the presence of alkali metal colloids associated with halogen bubbles. The position and the evolution of those aggregate centre absorption bands induce us to consider the possibility of metallic hydrogen. This hypothesis seems verified in the case of LiF bombarded with protons.
\end{abstract}

1. Introduction. - Les irradiations d'halogénures alcalins avec des particules chargées énergétiques créent des défauts qui sont essentiellement de trojs types:

1) Défauts liés à l'ionisation.

2) Défauts dus aux chocs des particules avec les ions du réseau.

3) Défauts associés aux particules implantées.

Dans le présent travail nous étudions principalement les défauts créés dans la zone d'ionisation des particules c'est-à-dire dans la zone où la particule a une grande énergie. Le bombardement à la température ambiante de $\mathrm{LiF}$ et $\mathrm{KCl}$ avec des particules alpha de $56 \mathrm{MeV}$, deutons de $28 \mathrm{MeV}, \mathrm{Li}^{3+}$ de $28 \mathrm{MeV}, \mathrm{Cl}^{8+}$ de $67 \mathrm{MeV}$, révèle après irradiation des centres $F . F_{n}, V_{n}$ et des centres évoluant au cours du temps. Une partie de ces derniers centres est identifiée, il s'agit de $F_{2}^{+}$dans $L_{-i F}$ et $F_{3}^{+*}$ dans $\mathrm{KCl}$. L'évolution de ces défauts au cours du temps met en évidence des mécanismes ne faisant pas intervenir d'électrons. Il existe donc des pièges à électrons créés par les particules dans la zone où la perte d’énergie par ionisation est prédominante. Dans le cas de LiF il existe un défaut associé à l'interstitiel lithium que nous devons observer lors dirradiation a basse température, ce défaut constituant le piège à électrons le plus simple. Lexistence de tels délauts portant sur les cations du résealu est fortement probable étant donné que la perte d'énergie par choc à haute énergie représente environ un millième de la perte d'énergie électronique. Si de tels défauts existent, il est possible d'envisager qu'ils puissent s'agglomérer par des traitements thermiques et former des défauts ayant des caractéristiques collodiales.

2. Techniques expérimentales. - Les cibles utilisées sont obtenues à partir de blocs monocristallins de LiF et $\mathrm{KCl}$ provenant de "Quartz et Silice "; certaines sont clivées suivant $\{100\}$ et d'autres polies mécaniquement suivant $\{110\}$.

Les ions utilisés sont obtenus respectivement: - au synchrocyclotron de l'Institut de Physique Nucléaire de Lyon pour les alpha de $56 \mathrm{MeV}$ et deutons de $28 \mathrm{MeV}$;

- au tandem FN du Département de Physique des Basses Energies du CEN Saclay pour les $\mathrm{Li}^{3+}$ de $28 \mathrm{MeV}$ et $\mathrm{Cl}^{8+}$ de $67 \mathrm{MeV}$.

Les flux pour ces irradiations sont compris entre $10^{9}$ et $10^{11}$ particules par centimètre carré par seconde. Labsorption optique des cibles irradiées est mesurée avec un spectrophotomètre à double faisceau « Beckman DK 2 a ». Les traitements thermiques sont elfectués a lia pression atmosphérique, les températures sont comprises entre 100 et $\Delta n 0{ }^{\circ} \mathrm{C}$.

Lirradiation de cristaux à la température de razote liquide est réalisée avec un dispositif permet- 
tant successivement l'irradiation puis les mesures d'absorption optique sans réchauffement des échantillons [1].

3. Centres évoluant au cours du temps dans LiF. A la température ambiante, pour des doses d'ions inférieures à $10^{12}$ ions $/ \mathrm{cm}^{2}$, on observe uniquement des centres $F$ dont la cinétique de croissance pour un flux $\varphi$ donné est linéaire en fonction du temps dans un système de coordonnées logarithmiques $\left(N_{\mathrm{F}} / \mathrm{cm}^{2}=K \varphi t^{0 / 9}\right)$. Connaissant le nombre d'ions d'énergie $E$ injectés, on en déduit une énergie dissipée par $\mathrm{cm}^{2}$ donc une perte d'énergie moyenne nécessaire pour observer un centre $F$ et dans le cas de deutons on obtient $600 \mathrm{eV}$. Pour des doses totales comprises entre $10^{12}$ et $5 \times 10^{13}$ ions $/ \mathrm{cm}^{2}$, on observe des centres $F$, des centres $F_{2}$ et des centres ayant des bandes situées à $6250 \AA$ (Fig. 1) et $3200 \AA$ environ qui évoluent au cours du temps après irradiation.

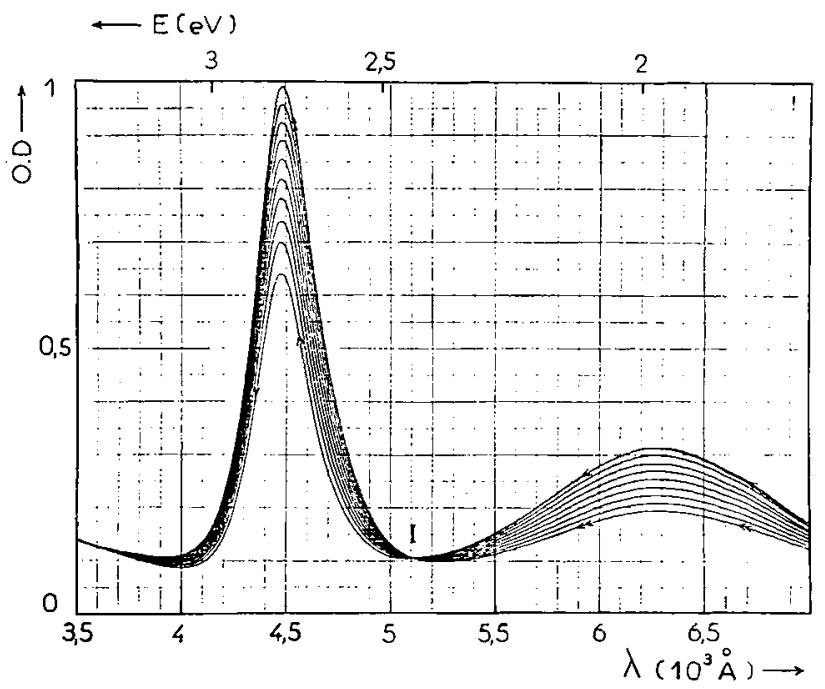

Fig. 1. - Evolution à la température ambiante du spectre d'absorption optique d'un cristal de LiF irradié avec $1012 \mathrm{~g} / \mathrm{cm}^{2}$. Les spectres sont tracés toutes les $10 \mathrm{~min}$.

$\rightarrow-$ premier spectre, $\rightarrow-$ dernier spectre.

Les caractéristiques de la bande d'absorption à $6250 \AA$ permettent de l'identifier comme étant la bande d'absorption du centre $\mathrm{F}_{2}^{+}$[2], [3]. Une précédente étude [4] montre que les centres $\mathrm{F}_{2}^{+}$se détruisent au profit des centres $\mathrm{F}_{3}^{+}$et que les mécanismes proposés [2], [5] quant à l'évolution des centres $F_{2}^{+}$ dans LiF ne s'appliquaient pas. Les électrons de neutralité de ces centres doivent être piégés par d'autres défauts et la concentration en centre $F$ est suffisamment importante pour que le processus $\mathrm{F}_{2}^{+} \rightarrow \mathrm{F}_{3}^{+}$se réalise.

3. 1 Etude des Centres $\mathrm{F}_{2-}^{+}$. - La création des centres $\mathrm{F}_{2}^{+}$est sensiblement indépendante du flux de particules, le rendement de création est plus important dans le cas d'une irradiation suivant une direction $\{110\}$.
La distribution des centres $\mathrm{F}_{2}^{+}$en fonction de la pénétration des particules est inhomogène (Fig. 2a). L'évolution de cette distribution au cours du temps (Fig 2b) montre d'une part que la vitesse de destruction des centres $\mathrm{F}_{2}^{+}$est fonction de la concentration initiale et d'autre part que la cinétique est du second ordre: le temps au bout duquel la moitié des centres $\mathrm{F}_{2}^{+}$a disparu est compris entre 85 et 200 min suivant la concentration initiale. Les cinétiques sont donc beaucoup plus rapides que celles obtenues avec des cristaux irradiés aux électrons [6].

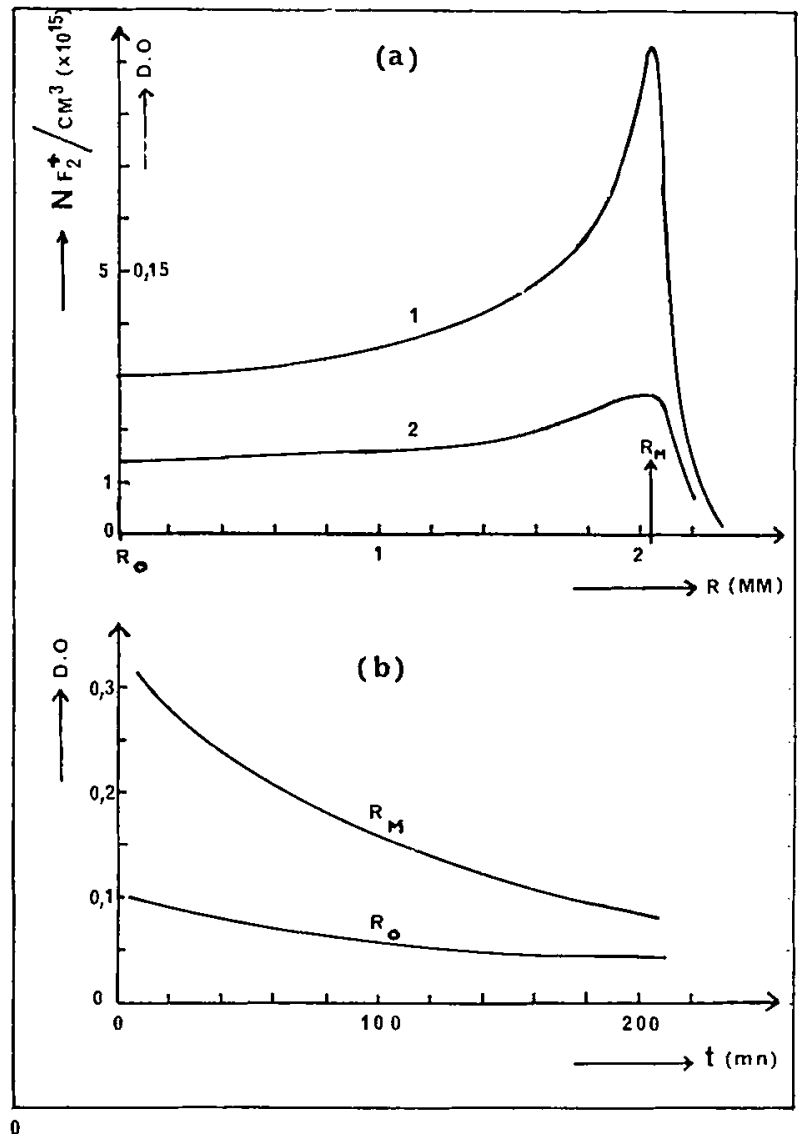

FIG. 2. - a) Distribution des centres $\mathrm{F}_{2}^{+}$en fonction de la pénétration des ions incidents dans un cristal de LiF irradié avec $10^{13}$ deutons $/ \mathrm{cm}^{2}$ à $300 \mathrm{~K}:$ 1) 20 nin. après irradiation ; 2) $200 \mathrm{~min}$. après irradiation. b) Evolution au cours du temps en deux points $R_{0}$ et $R_{M}$ où la concentration initiale des centres $\mathrm{F}_{2}^{+}$est différente.

Pour interpréter à la fois la création, l'évolution et les propriétés du centre $\mathrm{F}_{2}^{+}$, il faut considérer que Ja particule crée des défauts dans un volume limite autour de sa trajectoire et donc faire appel à une notion de gaine d'interaction.

La distribution des centres $F$ est inhomogène [7] et leur création est linéaire en fonction du temps tant qu'il n'y a pas d'interactions entre ces gaines c'est-à-dire jusqu'à des doses denviron $10^{12}$ ions $/ \mathrm{cm}^{2}$. ce qui implique un rayon d'action voisin de $50 \AA$. Les centres $\mathrm{F}_{2}^{+}$apparaissent à partit de cette dose; 
ils se trouvent donc situés dans des régions où la concentration en centres $\mathrm{F}$ est très élevée. L'interaction entre les centres $\mathrm{F}_{2}^{+}$et les centres $\mathrm{F}$ est donc un mécanisme probable, ce qui justifie le processus $\mathrm{F}_{2}^{+}+\mathrm{F} \rightarrow \mathrm{F}_{3}^{+}$.

3.2 Existence de pièges a Électrons, - Pour que le mécanisme $\mathrm{F}_{2}^{+}+\mathrm{F} \rightarrow \mathrm{F}_{3}^{+}$se réalise préférentiellement, il faut avoir créé, par irradiation, des pièges à électrons. Dans le cas de LiF un défaut associé à l'interstitiel lithium a été identifié par Farge [8], c'est le centre I qui possède une bande d'absorption à $5500 \AA$. Afin d'observer ces centres I nous avons irradié un cristal de $\mathrm{LiF}$ à la température de l'azote liquide. La figure (3) met en évidence la bande d'absorption des centres I pour un cristal irradié avec $10^{14}$ deutons $/ \mathrm{cm}^{2}$. Ceci confirme d'une

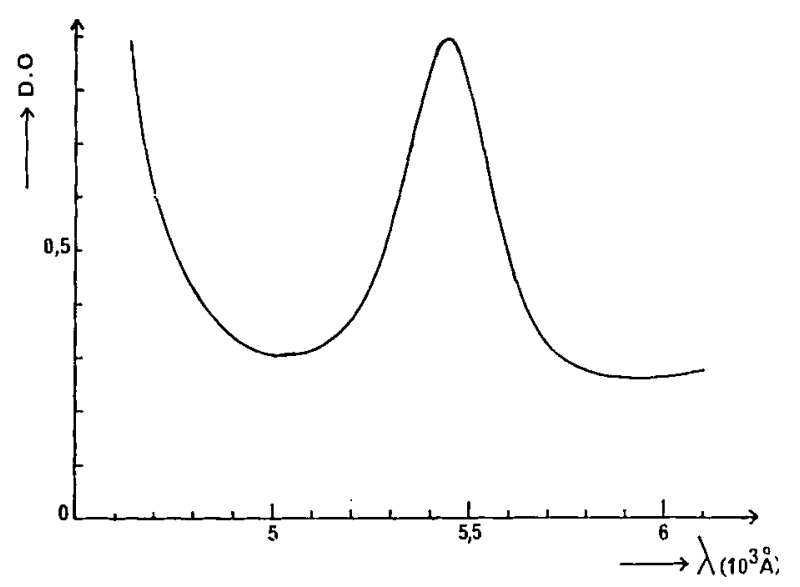

Fig. 3. - Bande d'absorption à $77 \mathrm{~K}$ des centres I dans LiF irradié avec 1014 deutons $/ \mathrm{cm}^{2}$ à $77 \mathrm{~K}$.

part l'existence d'interstitiels lithium créés par bombardement avec des particules chargées et d'autre part la création de pièges à électrons de nature cationique. La création de tels pièges est interprétable grâce aux collisions élastiques. La perte d'énergie nucléaire représente environ un millième de la perte d'énergie électronique à haute énergie. On peut par un calcul simple évaluer le nombre d'atomes déplacés par chocs. Puisqu'il est nécessaire en moyenne de céder une énergie de $600 \mathrm{eV}$ pour observer un centre $\mathrm{F}$ avec des deutons de $28 \mathrm{MeV}$, une énergie dissipée de $6 \times 10^{20} \mathrm{eV} / \mathrm{cm}^{3}$ correspond à une concentration de $10^{18}$ centres $\mathrm{F} / \mathrm{cm}^{3}$. En adoptant la valeur moyenne de $25 \mathrm{eV}$ évaluée par Seitz [9] pour placer un atome en position interstitielle, on obtient environ $10^{16}$ atomes déplacés par $\mathrm{cm}^{3}$, ce qui est de l'ordre de grandeur de la concentration des centres $\mathrm{F}_{2}^{+}$.

3.3 ETUDE DE LA BANIDE D'ABSORPTION SITUÉE A $3200 \AA$. - La bande à $3200 \AA$ n'a pas été identifiée à notre connaissance. Elle présente une amplitude plus importante dans le cas dirradiation suivant une direction $\{100\}$ que $\{110\}$ pour une même dose totale d'ions. L'influence de l'orientation ne peut être expliquée par un simple mécanisme d'ionisation, de plus, l'irradiation créant des défauts cationiques il doit donc exister des lacunes cationiques dans le réseau. Si l'on suppose que cette bande à $3200 \AA$ est associée à un défaut à base de lacune cationique, l'influence de l'orientation se justifie car la direction $\{110\}$ est une direction de canalisation plus importante que la direction $\{100\}$. De telles lacunes cationiques peuvent piéger des atomes halogènes et former des bulles élémentaires d'halogène. Cette bande située vers $3200 \AA$ est obtenue aussi par irradiation à la température de l'azote liquide avec des ions $\alpha$ ou deutons de plus faible énergie $(2 \mathrm{MeV})$. Elle se transforme en une bande située dans la région $M$, localisée à $4300 \AA$ et de largeur à mi-hauteur $0,3 \mathrm{eV}$.

4. Centres évoluant au cours du temps dans $\mathbf{K C l}$. L'irradiation de cristaux de $\mathrm{KCl}$ à $300 \mathrm{~K}$ avec $10^{13} \alpha$ / $\mathrm{cm}^{2}$ crée des défauts évoluant au cours du temps. Les évolutions étant très lentes et de faibles amplitudes, nous avons préféré utiliser des ions $\mathrm{Cl}^{8+}$ de $67 \mathrm{MeV}$. En effet, deux particules de même énergie totale mais de masse différente créent sensiblement le même nombre de défauts par ionisation, mais la concentration de ces défauts est plus importante pour les particules les plus lourdes. Si les centres qui évoluent ont des propriétés analogues aux centres $\mathrm{F}_{2}^{+}$dans LiF la vitesse de destruction sera augmentée dans le cas d'irradiation avec des ions $\mathrm{Cl}^{8+}$. Sur la figure 4 nous avons reporté les spectres optiques correspondant au début et à la fin de l'évolution; on observe trois bandes décroissant au cours du temps situées aux environs de $9500 \AA, 7500 \AA$ et $5400 \AA$, et une bande croissant dans la région $M$. La bande située à $9500 \AA$ est attribuée au centre $\mathrm{F}_{3}^{+}$[10]. Lors de la transformation d'un tel centre on n'observe pas d'augmentation dans la région correspondant aux bandes d'absorption $R_{1}$ et $R_{2}$, et dans ce cas, il y a encore absence d'électrons de neutralité.

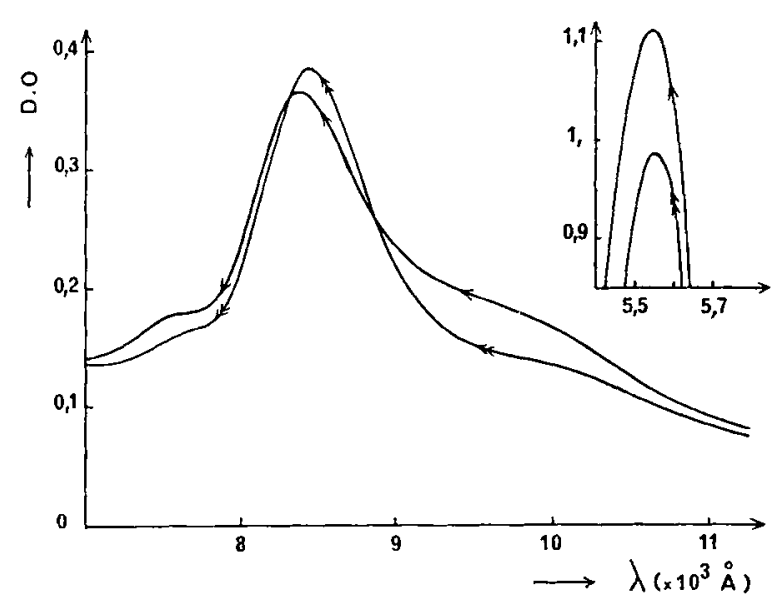

Fig. 4. - Evolution à $300 \mathrm{~K}$ du spectre d'absorption optique d'un cristal de $\mathrm{KCl}$ irradié avec $7 \times 10^{12} \mathrm{Cl}^{8+} / \mathrm{cm}^{2}$ $\rightarrow-15 \mathrm{~min}$. après irradiation; $\rightarrow-200 \mathrm{~min}$. après irradiation. 
Nous ne pouvons pas envisager un processus d'évolution des centres $\mathrm{F}_{3}^{+}$dans $\mathrm{KCl}$ analogue à celui obtenu pour $\mathrm{F}_{2}^{+}$dans LiF car la bande d'absorption du centre $\mathrm{F}_{4}^{+}$située à $1,67 \mu$ n'est pas observée. Par contre, on peut envisager un mécanisme tel que $\mathrm{F}_{3}^{+} \rightarrow \mathrm{F}_{2}+\square$ il y aurait donc libération d'une lacune anionique. Les bandes situées vers $5400 \AA$ et $7500 \AA$, mal résolues, peuvent être associées, comme dans le cas de $\mathrm{LiF}$, à des défauts d'origine cationique. Une telle hypothèse reste à préciser d'autant que l'irradiation à la température de l'azote liquide n'a pas permis de révéler une bande associée à l'interstitiel potassium.

5. Traitements thermiques. - Nous pouvions penser que les défauts cationiques, créés lors des irradiations, pouvaient s'agglomérer par traitement thermique. Dans ce but, nous avons irradié des cristaux avec des doses comprises entre $10^{14}$ et $10^{15}$ ions/ $\mathrm{cm}^{2}$ et effectué des recuits à des températures de l'ordre de $300{ }^{\circ} \mathrm{C}$ pour $\mathrm{LiF}$ et $200{ }^{\circ} \mathrm{C}$ pour $\mathrm{KCl}$. Ces traitements thermiques font disparaitre progressivement les bandes d'absorption $F$ et $F_{n}$ alors qu'il en subsiste deux dans chaque halogénure. Elles sont situées l'une dans le visible, l'autre dans l'ultraviolet; leurs amplitudes diminuent simultanément et elles se déplacent l'une vers l'autre (Fig. 5). Les

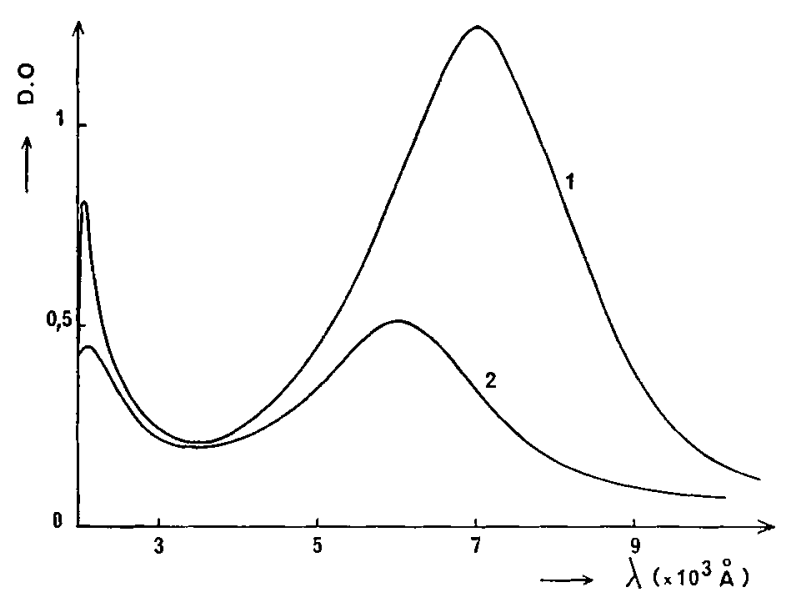

FIg. 5. - Evolution thermique du spectre d'absorption optique d'un cristal de $\mathrm{KCl}$ irradié avec $1 \mathrm{C}^{14}$ deutons $/ \mathrm{cm}^{2}$ à $300 \mathrm{~K}$. Spectre 1: Après recuit à $190^{\circ} \mathrm{C}$ pendant I $\mathrm{h}$. Spectre 2 : Après recuit à $190^{\circ} \mathrm{C}$ pendant $2 \mathrm{~h}$.

positions varient de $2150 \rightarrow 2300 \AA$ et $7000 \rightarrow 6000 \AA$ dans $\mathrm{KCl}$ alors que dans $\mathrm{LiF}$, elles varient de $2900 \rightarrow 3100 \AA$ et $4500 \rightarrow 4300 \AA$. Les bandes situées à $4500 \AA(\mathrm{LiF})$ et $7000 \AA(\mathrm{KCl})$ peuvent être associées à des petits collö̈des métalliques, par contre l'évolution avec la température ne peut être expliquée par la théorie de Mie [12]. La bande d'absorption UV dans $\mathrm{KCl}$ pourrait être associée à des centres $V_{2}$ ou $V_{3}$ mais dans le cas de LiF la bande située vers $2900 \AA$ ne correspond pas aux centres $V_{3}$. On peut cependant essayer de relier ces bandes d'absorption aux défauts créés par chocs. L'existence de défauts cationiques pouvant entraîner la formation de petits colloìdes métalliques et de bulles d'halogène dans la matrice (Fig. 6), l'évolution simultanée de ces colloïdes et de ces bulles semble indiquer un couplage entre les deux systèmes. Nous pouvons imaginer des colloïdes de métal situés à l'intérieur des bulles d'halogène c'est-à-dire sans contact avec la matrice. On remarque que plus l'ion alcalin est léger plus la bande d'absorption tend vers l'ultraviolet alors que plus l'ion halogène est léger, plus la bande d'absorption associée tend vers le visible. Il existerait donc une certaine convergence qui correspondrait à un élément ayant à la fois les caractères métallique et moléculaire. Cet élément devrait être l'hydrogène.

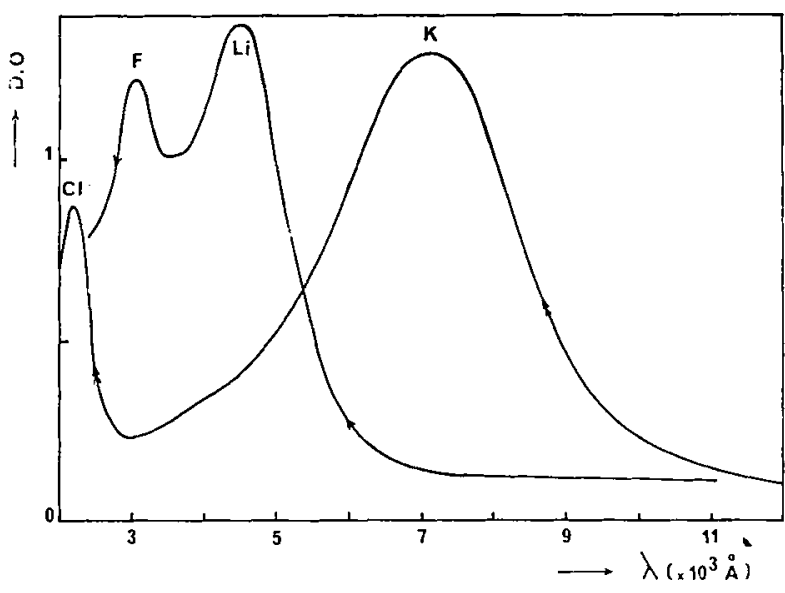

FIG. 6. - $\rightarrow$ - Spectre d'absorption optique d'un cristal de LiF irradié avec $10^{14}$ deutons $/ \mathrm{cm}^{2}$ à la température ambiante et traité thermiquement à $300{ }^{\circ} \mathrm{C}$ pendant $1 \mathrm{~h} . \rightarrow-$ Spectre d'absorption optique d'un cristal de $\mathrm{KCl}$ irradié comme précédemment et recuit à $190^{\circ} \mathrm{C}$ pendant $1 \mathrm{~h}$.

6. Essai de mise en évidence d'hydrogène à l'état métallique dans LiF irradié avec des protons. L'irradiation à la température de l'azote liquide avec $10^{16}$ protons $/ \mathrm{cm}^{2}$ d'une cible de LiF ne révèle pas immédiatement après irradiation une bande d'absorption pouvant être associée à l'hydrogène. Par contre un traitement thermique de $300^{\circ}$ pendant 15 min permet de meltre en évidence une bande d'absorption située aux environs de $3400 \AA$ (Fig. 7). Une précédente étude [13], [14] nous a permis de montrer que des ions alcalins $\mathrm{Li}^{+}, \mathrm{Na}^{+}$et $\mathrm{K}^{+}$implantés dans une matrice de Lif pouvaient s'agglomérer par traitements thermiques et former des colloïdes métalliques. Les positions des bandes d'absorption associées à ces colloïdes varient linéairement avec le paramètre du métal. Si l'on suppose que la bande d'absorption située à $3400 \AA$ est reliée à de l'hydrogène sous forme métallique [15], on peut en déduire son paramètre dans une matrice de $\mathrm{LiF}$ et on obtient $2,8 \AA$. Une étude théorique de J. J. Gilman [16] 


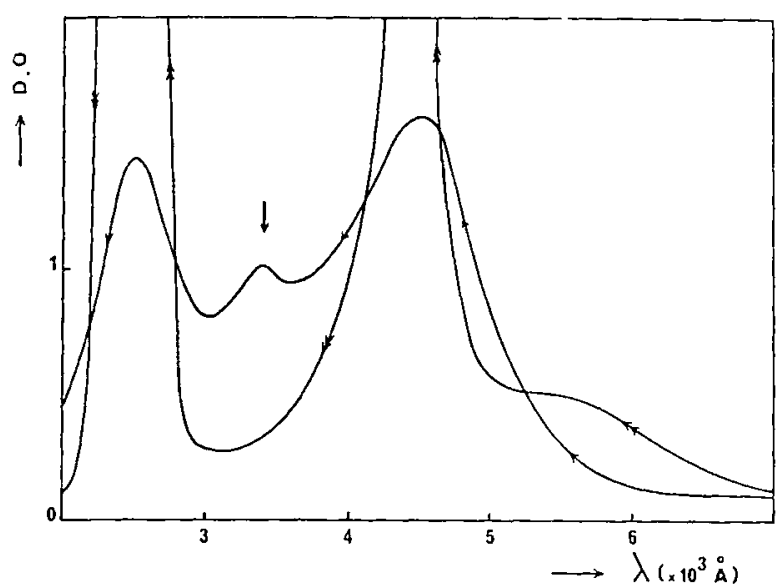

Fig. 7. -- Cristal de LiF irradié avec $10^{16}$ protons $/ \mathrm{cm}^{2}$ à $77 \mathrm{~K}$ $\rightarrow-$ Spectre à $300 \mathrm{~K}$.

$\rightarrow-$ Spectre après traitement thermique à $300^{\circ} \mathrm{C}$ pendant $15 \mathrm{~min}$.

a montré qu'un composé tel que $\mathrm{LiH}_{2} \mathrm{~F}$ pouvait être stable à la température ambiante et que l'hydrogène se trouverait sous une forme très condensée proche de l'état métallique. Utilisant les travaux de Wigner et Huntington [17], il a estimé à 2,86 $\AA$ le paramètre de l'hydrogène sous forme métallique dans LiF. Nous observons donc un bon accord entre la valeur théorique du paramètre et celle déduite de nos expériences.
Pour préciser ces résultats, il serait nécessaire d'effectuer des mesures de RPE et RMN.

7. Conclusion. -- L'évolution des centres chargés créés dans LiF et $\mathrm{KCl}$ par bombardement ionique de haute énergie à la température ambiante met en évidence la présence de pièges à électrons et conduit à envisager l'existence de défauts cationiques. Certains de ces défauts ont d’ailleurs été observés à basse température dans LiF (Centre 1). L'interaction de la particule incidente avec la cible se produit dans une gaine étroite et conduit à de grandes concentrations locales de défauts, ce qui permet d'expliquer les mécanismes de recombinaison observés $\left(\mathrm{F}_{2}^{+}+\mathrm{F} \rightarrow \mathrm{F}_{3}^{+}\right)$. Par traitements thermiques, ces défauts cationiques s'agglomèrent et font apparâtre des bandes collö̈dales. A partir des positions et de l'évolution de ces bandes, nous avons pu prévoir une bande liée à l'hydrogène sous une forme métallique dans LiF. Les irradiations de LiF avec des protons semblent confirmer cette hypothèse.

Remerciements. - Nous remercions M. le Prof. A. Sarazin et M. le Prof. M. Dufay pour leur assistance avec les accélérateurs Van de Graaff. Nous remercions également $M$. Delaunay pour toute l'aide qu'il nous a apportée lors des irradiations au CEN de Saclay.

\section{Bibliographie}

[1] Gurraud, G., Diplôme d`Etudes Supérieures, Lyon (1972).

[2] FARGE, Y., Thèse, Paris (1967)

[3] Nahum, J. and Wiegand, D. A., Phys. Rev. 154 (1967) 817.

[4] Thévenard, P., Perez, A., Davenas, J. and Dupuy, C. H. S. Phys. Stat. Sol. (a) 9 (1972) 517 ; Phys. Stat. Sol. (a) 10 (1972) 67.

[5] Nahum, J., Phys. Rev. 158 (1967) 814.

[6] Kaufman, J. V. R. et Clark, C. D., J. Chem. Phys. 38 (1963) 1388.

[7] Perez, A., Thévenard, P., Davenas, J. and Dupuy, C. H. S., Phys. Stat. Sol. (a) 18 (1973).

[8] Farge, Y., Lambert, M. et Guinier, A., J. Phys. Chem. Sol. 27 (1966) 499.
[9] SeItZ, F., Discussions Faraday Society 5 (1949) 271.

[10] Schneider, I. and Rabin, H., Phys. Rev. Lett. 13 (1964) 690.

[11] Schneider, I. and Rabin, H., Phys, Rev, 140 (1965) A 1983.

[12] MiE, G., Am. Physik 25 (1908) 377.

[13] Davenas, J, Thèse de Spécialité, Lyon 1972.

[14] Davenas, J., Perfiz, A., Thévenard, P. and Dupuy, C. H. S., Phys. Stat. Sol. (a) à paraître.

[15] Thévenard, P., Perez, A. et Dupuy, C. H. S., $C . R$. Hebd. Séan. Acad. Sci., Paris, à paraître.

[16] Gilman, J. J., Phys. Rev. Lett. 26 (1971) 546.

[17] Wigner, E. and Huntington, H. B., J. Chem. Phys. 3 (1935) 764. 